\title{
Statin use and high-dose statin use after ischemic stroke in the UK: a retrospective cohort study
}

This article was published in the following Dove Press journal: Clinical Epidemiology

\author{
Zhirong Yang' \\ Duncan Edwards' \\ Efthalia Massou' \\ Catherine L Saunders' \\ Carol Brayne ${ }^{2}$ \\ Jonathan Mant' \\ 'Primary Care Unit, Department of \\ Public Health and Primary Care, School \\ of Clinical Medicine, University of \\ Cambridge, Cambridge, UK; ${ }^{2}$ Institute of \\ Public Health, School of Clinical \\ Medicine, University of Cambridge, \\ Cambridge, UK
}

Background: Trial evidence supports statin use after ischemic stroke and recent American, European and British guidelines recommend high-intensity statins for this indication. Limited data are available describing current statin use among these patients in unselected settings. We conducted a cohort study to examine secular trends and factors associated with statin use and dose following ischemic stroke.

Methods: A retrospective cohort study of patients with first ischemic stroke between 2000 and 2014 was conducted using the Clinical Practice Research Datalink (CPRD). Proportions of statin users and high-intensity statin users within 2 years after stroke were estimated for each calendar year. We used Cox regression models to explore potential factors associated with statin use and Poisson regression models to calculate risk ratios for the use of a highintensity statin.

Results: A total of 80,442 patients with first stroke were analyzed. The proportion using statins within 2 years after stroke increased from $25 \%$ in 2000 to $70 \%$ in 2006 and remained at about $75 \%$ through 2014 . Among post-stroke statin users, high-intensity use accounted for approximately $15 \%$ between 2004 and 2011 and then increased to almost $35 \%$ in 2014 . Older patients (aged $\geq 75$ years), younger patients $(<45$ years), patients with no prior statin treatment, dementia, underweight, or absence of cardiovascular factors (coronary heart disease, smoking, obesity, diabetes, hypertension, or transient ischemic attack) were less likely to use statins and less likely to receive a high-intensity statin.

Conclusion: There has been an increase over time in both statin use and dose, but many patients with ischemic stroke continue to be under-treated. Clinical trials and policy interventions to improve appropriate post-stroke statin use should focus on younger and older patients, patients with no pre-stroke statin treatment, and patients without additional cardiovascular risk factors.

Keywords: cerebral ischemia, lipid-lowering, trend, factors

\section{Introduction}

Stroke remains one of the leading causes of death and disability in the UK and worldwide. ${ }^{1,2}$ While incidence and mortality rates are declining in developed countries such as the UK and the USA, related to improvements in health care and public health, post-stroke survival is also increasing, expanding the population in need of effective secondary prevention of recurrent strokes and cardiovascular events. $^{3,4}$ Ischemic stroke accounts for $80-90 \%$ of stroke in the UK, ${ }^{5}$ and statins can reduce the risk of stroke and cardiovascular events in these patients. ${ }^{6}$

Evidence supporting the benefits of statins after ischemic stroke has come in particular from two large-scale randomized controlled trials, the Heart Protection Study $(\mathrm{HPS})^{7}$ published in 2004 and the Stroke Prevention by Aggressive
Correspondence: Zhirong Yang

Primary Care Unit, University of

Cambridge, 2 Worts' Causeway,

Cambridge, CBI 8RN, UK

Tel +44 1223762539

Email zy266@medschl.cam.ac.uk 
Reduction in Cholesterol Levels (SPARCL) ${ }^{8}$ in 2006. This evidence is reflected in clinical guidelines, published since 2011, which now recommend high-intensity statin therapy be initiated or continued as first-line treatment in both women and men who have ischemic stroke, unless contraindicated. ${ }^{9-11}$ In parallel, the affordability and availability of high-intensity statins was increased as the patent for atorvastatin expired in 2011. Finally, a UK-specific event that may have increased post-stroke statin use is the Quality and Outcomes Framework (QOF) incentive to encourage family doctors to ensure ischemic stroke survivors maintain a total cholesterol target of $5 \mathrm{mmol} / \mathrm{L}$ or less, which was introduced in 2004 and continued over the next 10 years. ${ }^{12}$

Statin therapy is therefore now an established key component of secondary prevention after ischemic stroke.${ }^{13,14}$ Some previous studies have explored persistence with statin therapy in the initial years after a stroke, and have suggested different factors that are associated with nonpersistence, including age, polypharmacy, and comorbidity; most studies are not of sufficient size to explore all key factors, and it is also likely that patterns of use have changed over time. ${ }^{15-17}$ There are few recent published data on trend in and factors for statin use and dose after ischemic stroke.

This study examines how statin prescribing after stroke has changed over time between 2000 and 2014 in a large representative sample of the UK general population, and explores factors associated with both statin use and with high-intensity statin use.

\section{Methods}

\section{Data source}

We conducted a retrospective cohort study using the Clinical Practice Research Datalink (CPRD), which provides anonymized data extracted from primary care medical records (including community prescribing information), with coverage of a representative sample of approximately $7 \%$ of the UK population from more than 670 practices. ${ }^{18}$

\section{Study population}

We examined the whole history of patients with any stroke codes recorded in the CPRD and included patients aged at least 18 years with a diagnosis of first stroke between 1 January 2000 and 31 December 2014. The study population comprises patients registered with participating general practices, including nursing home patients. Patients with any Read codes relating to hemorrhagic stroke for the index stroke were excluded. To ensure the quality of recording of pre-existing diagnoses and medications, eligible patients were also required to have at least 12-month record information before the index date. Codes for stroke were listed in Table S1.

\section{Statin use after stroke}

Statins were identified using British National Formulary $\operatorname{codes}^{18}$ within 2 years after the index stroke (Table S2). All the statins which had ever been on the market in the UK were analyzed in this study, including atorvastatin, cerivastatin, fluvastatin, pravastatin, rosuvastatin, and simvastatin. Statin use was defined as the first prescription of statins on or after the index date. According to the British NICE guideline,${ }^{11}$ high-intensity statin use was defined as atorvastatin $\geq 20 \mathrm{mg} /$ day, rosuvastatin $\geq 10 \mathrm{mg} /$ day, and simvastatin $80 \mathrm{mg} /$ day, medium-intensity as atorvastatin $10 \mathrm{mg}$, fluvastatin $80 \mathrm{mg}$, rosuvastatin $5 \mathrm{mg}$, simvastatin 20 and $40 \mathrm{mg}$, and low-intensity as others. Post-stroke statin intensity was defined based on the highest intensity used within 2 years after stroke. Accordingly, statin users were further classified into high-intensity statin users and low/medium-intensity users. When investigating the use of high-intensity statins, we excluded cerivastatin, which was withdrawn from the world market in 2001 due to adverse effects.

\section{Covariates}

Demographic covariates included age, gender, and socioeconomic status. Age was calculated on the date of index stroke and categorized into six groups (18-44, 45-54, 55$64,65-74,75-84$, and $\geq 85$ years). Index of Multiple Deprivation (IMD) grouped by quintile was used as an indicator of socioeconomic status. The IMD includes seven domains: income; employment; health and disability; education, skills and training; barriers to housing and services; crime; and living environment. ${ }^{19}$ Where patient-level IMD was missing, we used the practice-level IMD instead. Prior medical condition was defined as the presence of any relevant code before the index stroke date. These conditions included atrial fibrillation (AF), cancer, coronary heart disease (CHD), chronic kidney disease (CKD), chronic liver disease (CLD), chronic obstructive pulmonary disease (COPD), dementia, diabetes, heart failure, hypertension, myopathy, peripheral artery disease (PAD), and transient ischemic attack (TIA), which are conditions considered to potentially influence statin use. ${ }^{20}$ The code lists for these conditions were developed as part of a project describing 
multimorbidity in primary care ${ }^{21}$ and are publicly available on the website: http://www.phpc.cam.ac.uk/pcu/cprd_cam/ codelists/. Smoking (current, ex-, and never smoking) and body mass index (BMI $<18.5,18.5-24,25-29,30-34$, and 35 or more) were measured using the most recent data before the index stroke. Year of stroke was categorized into three periods (2000-2006, 2007-2010, and 20112014) according to the publication time of the landmark trials $^{7,8}$ and guidelines. ${ }^{9-11}$

Patients with any statin prescriptions during the 365 days prior to their index stroke were regarded as pre-stroke statin users, with the intensity defined as highest intensity they ever used in this period. The same time window was used to define other lipid-lowering treatment (LLT) before stroke. For long-term conditions, an absence of related codes was regarded as an absence of the condition.

\section{Statistical analysis}

Baseline characteristics and statin use patterns after stroke were described for the overall stroke cohort. We also examined the similarity in the baseline characteristics and the statin use patterns between our overall cohort and the subgroup of patients with specific ischemia Read codes in order to verify that our practical definition of ischemic stroke was robust. We calculated the percentage of post-stroke statin use by year of stroke. Patient characteristics were described for statin users and non-users.

We estimated average hazard ratios (HRs) for statin use during the 2-year follow-up using Cox proportional hazards models with robust standard errors that allow for intragroup correlation to account for possible clustering effects by general practice. Follow-up started from the index stroke until the first statin prescription (except for the analysis of statin dose) after stroke or censoring, whichever occurred earlier. Censoring included death, emigration from the CPRD, or the end of the follow-up period. HRs were calculated in an unadjusted model, in a partial adjustment model that accounted for demographics, and in a full model that simultaneously adjusted for demographics, prior conditions, smoking, BMI, and period of stroke.

Considering high dose statin use, we investigated the change in the proportion of high-intensity statin prescription over time and described patient characteristics for high-intensity and low/medium-intensity statin users. Restricting analysis to statin users who completed 2-year follow-up after stroke, we used modified Poisson regression with robust standard error to explore possible factors for high-intensity statin treatment. ${ }^{22}$ Risk ratios (RRs) were calculated in an unadjusted model, in a partial adjustment model, and in a full model.

Based on the HR or RR estimated from a full model, we then calculated relative percentage reduction for potential factors associated with decreased use of statins and a high-intensity statin.

All the data management and statistical analyses were conducted using Stata 15. Quality control was performed before analysis (Table S3).

We reported the results according to the RECORD statement (Supplemental Material).

\section{Sensitivity analysis}

We conducted a series of sensitivity analysis to examine the robustness of the study results. 1) A time-partitioned analysis was conducted to accommodate potential non-proportionality. In this analysis, the follow-up period was partitioned at 90 days after stroke, based on the inspection of the log-log plots and considering 90-day mortality or disability as a key outcome assessed in trials and observational studies of statin therapy after stroke. $^{23}$ 2) We restricted the analysis to patients who had specific codes for ischemic stroke in the CPRD (ie, excluding any unspecified stroke). 3) We only included patients with no prior statin treatment before stroke (ie, excluding prevalent statin users). 4) To evaluate possible impact of missing data on the results, we restricted the analysis to those with individual IMD. We carried out an extreme-case sensitivity analysis by changing the missing value of smoking status to never smoking or current smoking and changing the missing value of BMI to the 5 th or 95 th percentile value, respectively.

\section{Ethics approval and patient consents}

Ethics approval was obtained from the Independent Scientific Advisory Committee of the CPRD (protocol number 17_012R), with no written consent from participants required.

\section{Results}

\section{Characteristics of the stroke cohort}

The analysis included 80,442 patients with a first stroke, among whom 26,339 had specified ischemic stroke codes (Figure 1). Overall, 63.8\% patients $(51,338)$ ever used statins within 2 years after stroke, among whom 42.7\% (21,934/ 51,338 ) were on statins prior to stroke. Of the 51,338 patients, 51,326 had specified codes for statin intensity classification and were included in the analysis for high-intensity statin use. Characteristics of the stroke cohort by statin use are shown in 


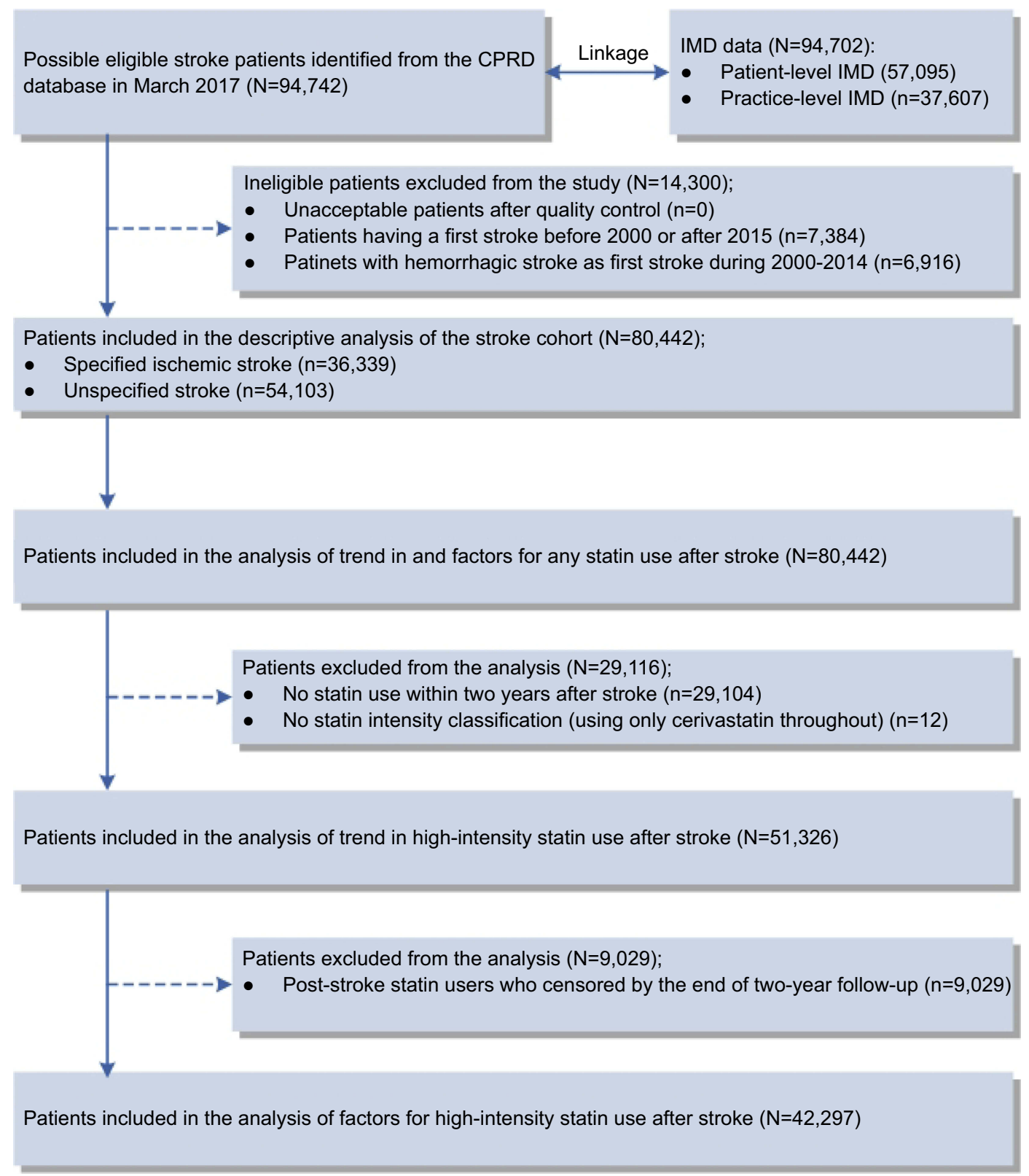

Figure I Flowchart of study population inclusion.

Abbreviations: CPRD, Clinical Practice Research Datalink; IMD, Index of Multiple Deprivation.

Table 1. These characteristics were similar between specified ischemic and unspecified stroke (Table S4).

Among statin users, most were commenced on treatment within 90 days after stroke (84.3\%) with a mediumintensity dose (73.8\%). Those who did not change initial intensity through 2 years accounted for $86.4 \%$. These statin use patterns were similar between patients with specified ischemic stroke and unspecified stroke (Table S5). Only $3.9 \%$ of no statin users $(1,128 / 29,104)$ were prescribed alternative lipid-lowering drugs within a 2 -year period after stroke.

\section{Trend in statin use and dose after stroke}

Overall, the percentage of statin use increased steadily between 2000 and 2006, from 25\% to $70 \%$ (Figure 2). While the percentage continued to grow slightly afterward, it became stable at about $75 \%$ through 2014 . Two factors accounted for the trend in increasing statin use. First, the general trend ran parallel to an increasing trend in statin use prior to stroke, which accounted for less than $10 \%$ of patients in 2000 and over $40 \%$ in 2014, and these prior users of statins continued to use them after stroke in almost $90 \%$ of cases throughout 2000-2014. Second, the use of statins among pre- 
Table I Characteristics of the stroke cohort by statin use after stroke

\begin{tabular}{|c|c|c|c|c|c|c|c|c|c|c|}
\hline \multirow{3}{*}{$\begin{array}{l}\text { Factor } \\
\text { Demographics }\end{array}$} & \multicolumn{6}{|c|}{ Statin use } & \multirow{2}{*}{\multicolumn{2}{|c|}{$\begin{array}{l}\text { No statin use } \\
(n=29,104)\end{array}$}} & \multirow{2}{*}{\multicolumn{2}{|c|}{$\begin{array}{l}\text { Overall } \\
(N=80,442)\end{array}$}} \\
\hline & \multicolumn{2}{|c|}{$\begin{array}{l}\text { High inten- } \\
\text { sity* } \\
(n=I \mid, 877)\end{array}$} & \multicolumn{2}{|c|}{$\begin{array}{l}\text { Low/Medium } \\
\text { intensity* } \\
(n=39,449)\end{array}$} & \multicolumn{2}{|c|}{$\begin{array}{l}\text { Any statin } \\
(n=5 I, 338)\end{array}$} & & & & \\
\hline & & & & & & & & & & \\
\hline Age (\%) & & & & & & & & & & \\
\hline Mean (SD) & 69.3 & $(11.6)$ & 72.3 & $(12.1)$ & 71.6 & $(12.1)$ & 79.0 & $(13.1)$ & 74.3 & (I2.9) \\
\hline 18-44 years & 274 & $(2.3)$ & 945 & $(2.4)$ & 1,219 & $(2.4)$ & 810 & $(2.8)$ & 2,029 & $(2.5)$ \\
\hline $45-54$ & 1,096 & $(9.2)$ & 2,539 & (6.4) & 3,636 & (7.1) & 900 & $(3.1)$ & 4,536 & $(5.6)$ \\
\hline $55-64$ & 2,499 & $(21.0)$ & 6,036 & $(I 5.3)$ & 8,536 & $(16.6)$ & I,799 & $(6.2)$ & 10,335 & (I2.9) \\
\hline $65-74$ & 3,639 & $(30.6)$ & 10,677 & $(27.1)$ & 14,322 & $(27.9)$ & 4,301 & $(14.8)$ & 18,623 & (23.2) \\
\hline $75-84$ & 3,410 & $(28.7)$ & 13,418 & $(34.0)$ & 16,832 & $(32.8)$ & 9,954 & $(34.2)$ & 26,786 & (33.3) \\
\hline$\geq 85$ & 959 & $(8.1)$ & 5,834 & $(14.8)$ & 6,793 & $(13.2)$ & 11,340 & (38.9) & 18,133 & (22.5) \\
\hline Female (\%) & 5,486 & $(46.2)$ & 18,594 & $(47.1)$ & 24,084 & $(46.9)$ & 17,252 & (59.3) & 41,336 & $(51.4)$ \\
\hline \multicolumn{11}{|l|}{$\mathrm{IMD}^{\mathrm{a}}(\%)$} \\
\hline Group I & 2,353 & $(19.8)$ & 8,350 & $(21.2)$ & 10,706 & $(20.9)$ & 6,125 & $(2 \mathrm{I} .1)$ & $|6,83|$ & (20.9) \\
\hline Group 2 & 2,361 & $(19.9)$ & 7,661 & $(19.4)$ & 10,023 & $(19.5)$ & 5,964 & $(20.5)$ & 15,987 & (19.9) \\
\hline Group 3 & 2,321 & $(19.5)$ & 8,158 & $(20.7)$ & 10,483 & $(20.4)$ & 6,126 & $(2 I . I)$ & 16,609 & (20.7) \\
\hline Group 4 & 2,456 & $(20.7)$ & 7,801 & $(19.8)$ & 10,259 & $(20.0)$ & 5,278 & $(18.1)$ & 15,537 & $(19.3)$ \\
\hline Group 5 & 2,386 & $(20.1)$ & 7,469 & $(18.9)$ & 9,857 & $(19.2)$ & 5,585 & $(19.2)$ & 15,442 & $(19.2)$ \\
\hline \multicolumn{11}{|l|}{ Prior conditions (\%) } \\
\hline $\mathrm{AF}$ & $\mathrm{I}, 537$ & $(12.9)$ & 6,018 & $(15.3)$ & 7,556 & $(14.7)$ & 5,612 & $(19.3)$ & 13,168 & $(16.4)$ \\
\hline Cancer & 987 & $(8.2)$ & 3,602 & $(9.1)$ & 4,590 & $(8.9)$ & 3,351 & $(\mathrm{II} .5)$ & 7,941 & $(9.9)$ \\
\hline CHD & 3,533 & $(29.8)$ & 7,645 & $(19.4)$ & 11,800 & $(21.8)$ & 5,320 & $(18.3)$ & 16,500 & (20.5) \\
\hline CKD & 259 & $(2.2)$ & 631 & $(1.6)$ & 891 & $(1.7)$ & 357 & $(1.2)$ & I,248 & $(1.6)$ \\
\hline CLD & 74 & $(0.6)$ & 273 & $(0.7)$ & 347 & $(0.7)$ & 271 & $(0.9)$ & 618 & $(0.8)$ \\
\hline COPD & 949 & $(8.0)$ & 2,970 & $(7.5)$ & 3,920 & $(7.6)$ & 2,387 & $(8.2)$ & 6,307 & $(7.8)$ \\
\hline Dementia & 183 & $(1.5)$ & 1,025 & $(2.6)$ & 1,208 & $(2.4)$ & 3,030 & $(10.4)$ & 4,238 & $(5.3)$ \\
\hline Diabetes & 2,807 & $(23.6)$ & 6,243 & $(15.8)$ & 9,052 & $(17.6)$ & 3,818 & $(13.1)$ & 12,870 & $(16.0)$ \\
\hline Heart failure & 832 & $(7.0)$ & 2,328 & $(5.9)$ & 3,161 & $(6.2)$ & 3,656 & $(12.6)$ & 6,817 & $(8.5)$ \\
\hline Hypertension & 6,837 & $(57.6)$ & 20,725 & $(52.5)$ & 27,567 & $(53.7)$ & 13,590 & $(46.7)$ & 41,157 & $(51.2)$ \\
\hline Myopathy & 691 & $(5.8)$ & 2,066 & $(5.2)$ & 2,757 & (5.4) & $\mathrm{I}, 577$ & $(5.4)$ & 4,334 & $(5.4)$ \\
\hline PAD & 1,019 & $(8.6)$ & 2,291 & $(5.8)$ & 3,310 & $(6.5)$ & 1,896 & $(6.5)$ & 5,206 & $(6.5)$ \\
\hline TIA & $\mathrm{I}, 7 \mid 4$ & $(14.4)$ & 5,277 & $(13.4)$ & 6,992 & $(13.6)$ & 4,180 & $(14.4)$ & 11,172 & $(13.9)$ \\
\hline \multicolumn{11}{|l|}{ Smoking status ${ }^{\mathrm{b}}(\%)$} \\
\hline Current-smoker & 2,889 & $(24.3)$ & 8,208 & $(20.8)$ & 11,101 & $(21.6)$ & 4,482 & $(15.4)$ & 15,583 & $(19.4)$ \\
\hline Ex-smoker & 3,950 & $(33.3)$ & 12,186 & $(30.9)$ & 16,137 & $(31.4)$ & 6,632 & $(22.8)$ & 22,769 & (28.3) \\
\hline Non-smoker & 4,807 & $(40.5)$ & 17,638 & $(44.7)$ & 22,450 & $(43.7)$ & 14,838 & $(51.0)$ & 37,288 & (46.4) \\
\hline \multicolumn{11}{|l|}{ BMI $^{c}(\%)$} \\
\hline Mean (SD) & 28.1 & $(5.3)$ & 27.1 & $(5.2)$ & 27.4 & $(5.2)$ & 25.8 & $(5.2)$ & 26.8 & $(5.3)$ \\
\hline$<18.5$ & 142 & $(1.2)$ & 784 & $(2.0)$ & 927 & $(1.8)$ & 1,093 & $(3.8)$ & 2,021 & $(2.5)$ \\
\hline $18.5-24$ & 3,022 & $(25.4)$ & 11,717 & $(29.7)$ & 14,742 & $(28.7)$ & 9,231 & $(3 \mid .7)$ & 23,973 & $(29.8)$ \\
\hline $25-29$ & 4,419 & $(37.2)$ & 13,525 & $(34.3)$ & 17,946 & $(35.0)$ & 7,498 & $(25.8)$ & 25,444 & (31.6) \\
\hline $30-34$ & 2,192 & $(18.5)$ & 5,938 & $(15.0)$ & 8,132 & $(15.8)$ & 2,697 & $(9.3)$ & 10,829 & $(13.5)$ \\
\hline$\geq 35$ & 1,079 & $(9.1)$ & 2,481 & $(6.3)$ & 3,560 & $(6.9)$ & 1,046 & $(3.6)$ & 4,607 & $(5.7)$ \\
\hline \multicolumn{11}{|l|}{ Medications before stroke (\%) } \\
\hline Statins $^{d}$ & 7,019 & $(59.1)$ & $14,9 \mid 2$ & $(37.8)$ & 21,934 & $(42.7)$ & 3,170 & $(10.9)$ & 25,104 & $(31.2)$ \\
\hline High & 4,327 & $(36.4)$ & 459 & $(1.2)$ & 4,786 & $(9.3)$ & 553 & $(1.9)$ & 5,339 & $(6.6)$ \\
\hline
\end{tabular}


Table I (Continued).

\begin{tabular}{|c|c|c|c|c|c|c|c|c|c|c|}
\hline \multirow[t]{2}{*}{ Factor } & \multicolumn{6}{|c|}{ Statin use } & \multirow{2}{*}{\multicolumn{2}{|c|}{$\begin{array}{l}\text { No statin use } \\
(n=29,104)\end{array}$}} & \multirow{2}{*}{\multicolumn{2}{|c|}{$\begin{array}{l}\text { Overall } \\
(N=80,442)\end{array}$}} \\
\hline & \multicolumn{2}{|c|}{$\begin{array}{l}\text { High inten- } \\
\text { sity* } \\
(n=I I, 877)\end{array}$} & \multicolumn{2}{|c|}{$\begin{array}{l}\text { Low/Medium } \\
\text { intensity* } \\
(n=39,449)\end{array}$} & \multicolumn{2}{|c|}{$\begin{array}{l}\text { Any statin } \\
(n=51,338)\end{array}$} & & & & \\
\hline Low/Medium & 2,686 & $(22.6)$ & 14,422 & (36.6) & 17,108 & (33.3) & 2,608 & $(9.0)$ & 19,716 & (24.5) \\
\hline Other LLT & 627 & (5.3) & 597 & (I.5) & 1,224 & $(2.4)$ & 556 & (1.9) & $\mathrm{I}, 780$ & $(2.2)$ \\
\hline
\end{tabular}

Notes: *Twelve patients only using cerivastatin were excluded out of the analysis of statin intensity, for which statin intensity classification is not available.

${ }^{\mathrm{a}} \mathrm{A}$ total of $36(0.04 \%)$ patients had missing value of IMD: $0(0.00), 10(0.03 \%), 10(0.02 \%)$, and $26(0.09 \%)$ for high intensity, low/medium intensity, any statin and no statin use, respectively. Group I is the least deprived group. ${ }^{\mathrm{b}} \mathrm{A}$ total of $4,802(6.0 \%)$ patients had missing value of smoking status: $23 \mathrm{I}$ (I.9\%), I,4I7 (3.6\%), I,650 (3.2\%), and 3,152 (10.8\%) for high intensity, low/medium intensity, any statin and no statin use, respectively. ${ }^{\mathrm{C}} \mathrm{A}$ total of 13,570 (16.9\%) patients had missing value of BMI: I,023 (8.6\%), 5,004 ( $12.7 \%), 6,03 \mathrm{I}$ (II.8\%), and 7,539 (25.9\%) for high intensity, low/medium intensity, any statin and no statin use, respectively. ${ }^{\mathrm{d}} \mathrm{A}$ total of 49 ( $\left.0.06 \%\right)$ patients had missing value of pre-stroke statin dose: $6(0.05 \%), 31(0.08 \%), 40(0.08 \%)$, and $9(0.03 \%)$ for high intensity, low/medium intensity, any statin and no statin use, respectively.

Abbreviations: AF, atrial fibrillation; BMI, body mass index; CHD, coronary heart disease; CKD, chronic kidney disease; CLD, chronic liver disease; COPD, chronic obstructive pulmonary disease; IMD, Index of Multiple Deprivation; LLT, lipid-lowering treatment; PAD, peripheral artery disease; SD, standard deviation; TIA, transient ischemic attack.

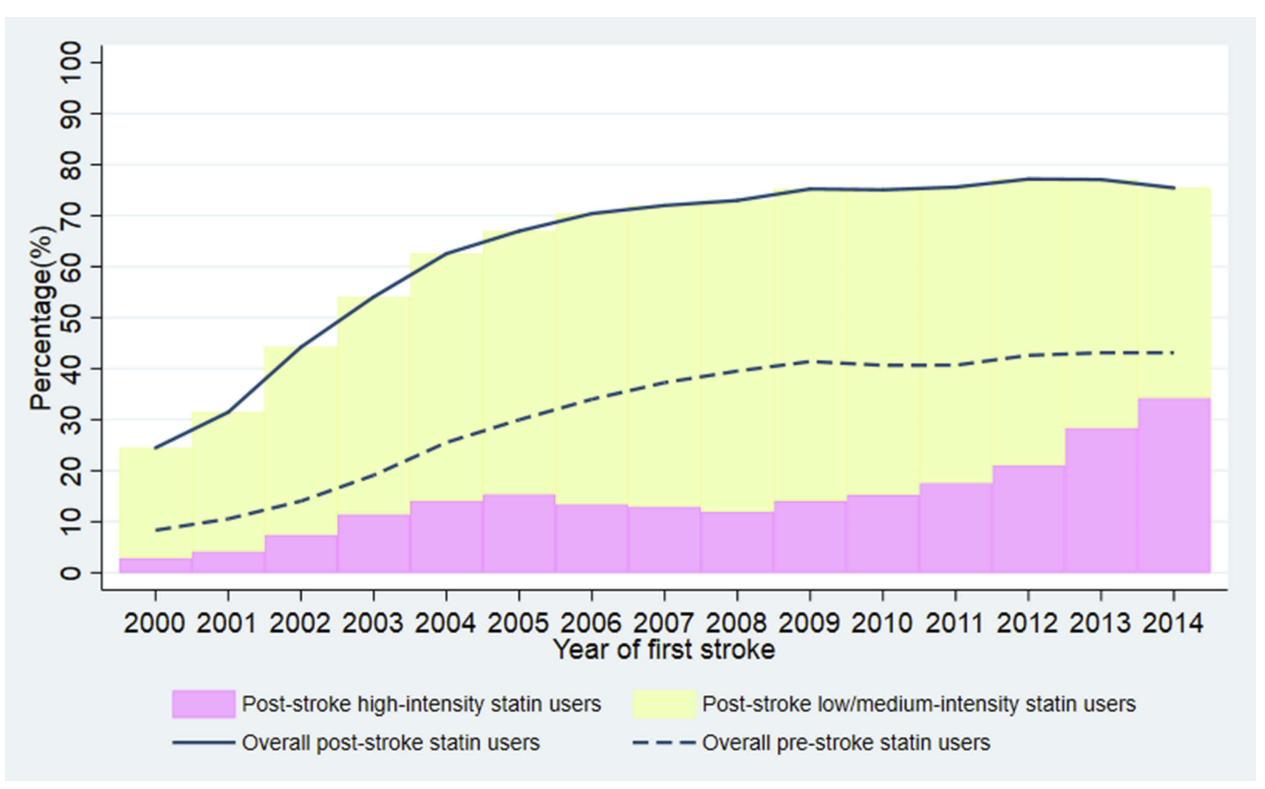

Figure 2 Trend in statin and intensity use after stroke.

stroke non-users increased from $20 \%$ to $60 \%$ between 2000 and 2006 (Figure 3).

Use of high-intensity statins has fluctuated over time, but there is evidence of an increase from $10 \%$ since 2008 , to about $35 \%$ in 2014 .

There were similar trends in the proportion of statin users and high-intensity statin users between specified ischemic and unspecified stroke, with slightly higher usage in the specified group for each calendar year (Figure S1).

\section{Factors associated with statin use and high-intensity statin use after stroke}

As shown in Table 2, in the fully adjusted model, extremes of age were associated with lower rates of all types of statin use, with people aged 18-44 years old and over 85 years old less likely than the 55-64-year-old age group to use statins (HR 0.52, 95\% CI 0.48-0.56, and HR 0.48, 95\% CI $0.46-0.50$, respectively). Statin usage was similar across all socioeconomic groups and between genders after adjustment.

Among prior conditions, CHD, TIA, hypertension, and diabetes had the strongest association with statin use, with any statin use between $8 \%$ and $22 \%$ higher after full adjustment. These conditions were also associated with highintensity statin use (final three columns in Table 2), from $8 \%$ to $58 \%$ higher than the relevant reference group in the full model. Notably, PAD, also an indication for statin use, was not associated with increased use of statins in the full 

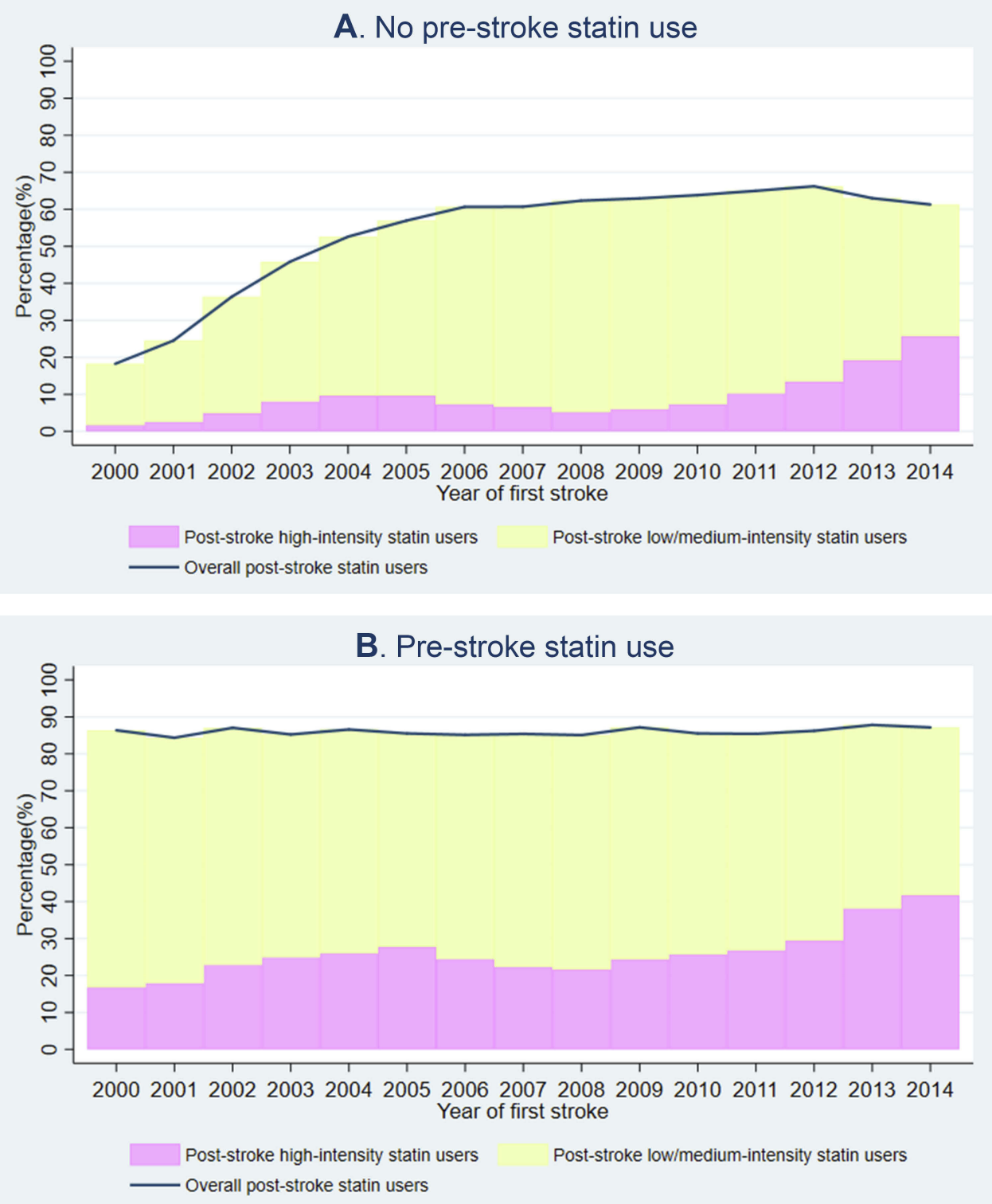

Figure 3 Trend in statin and intensity use after stroke by prior statin use $(\mathbf{A})$ no pre-stroke statin use; (B) pre-stroke statin use).

model, though it was associated with increased probability of high intensity of statins.

Smokers and obese patients likewise had significantly increased statin and high-intensity statin use.

In contrast, dementia, heart failure, and CLD were associated with the lowest statin use both before and after adjustment.

Previous use of statins was strongly associated with statin use after ischemic stroke, with an HR of $2.36(95 \%$ CI 2.30-2.42) in the fully adjusted model. Likewise, previous use of high-intensity statins was strongly associated with high-intensity statin use after ischemic stroke, with an
RR of 4.47 (95\% CI 4.51-4.99). Patients with pre-stroke non-statins LLT were less likely to use statins after ischemic stroke, but if they did use them, were more likely to receive a high intensity.

All the associations of factors with statin prescription and high dose use were categorized into three groups, in which patients may miss out the optimal statin treatment after stroke (Table 3).

\section{Sensitivity analysis}

The time partitioned analysis did not lead to important changes to the results with regard to what factors are 


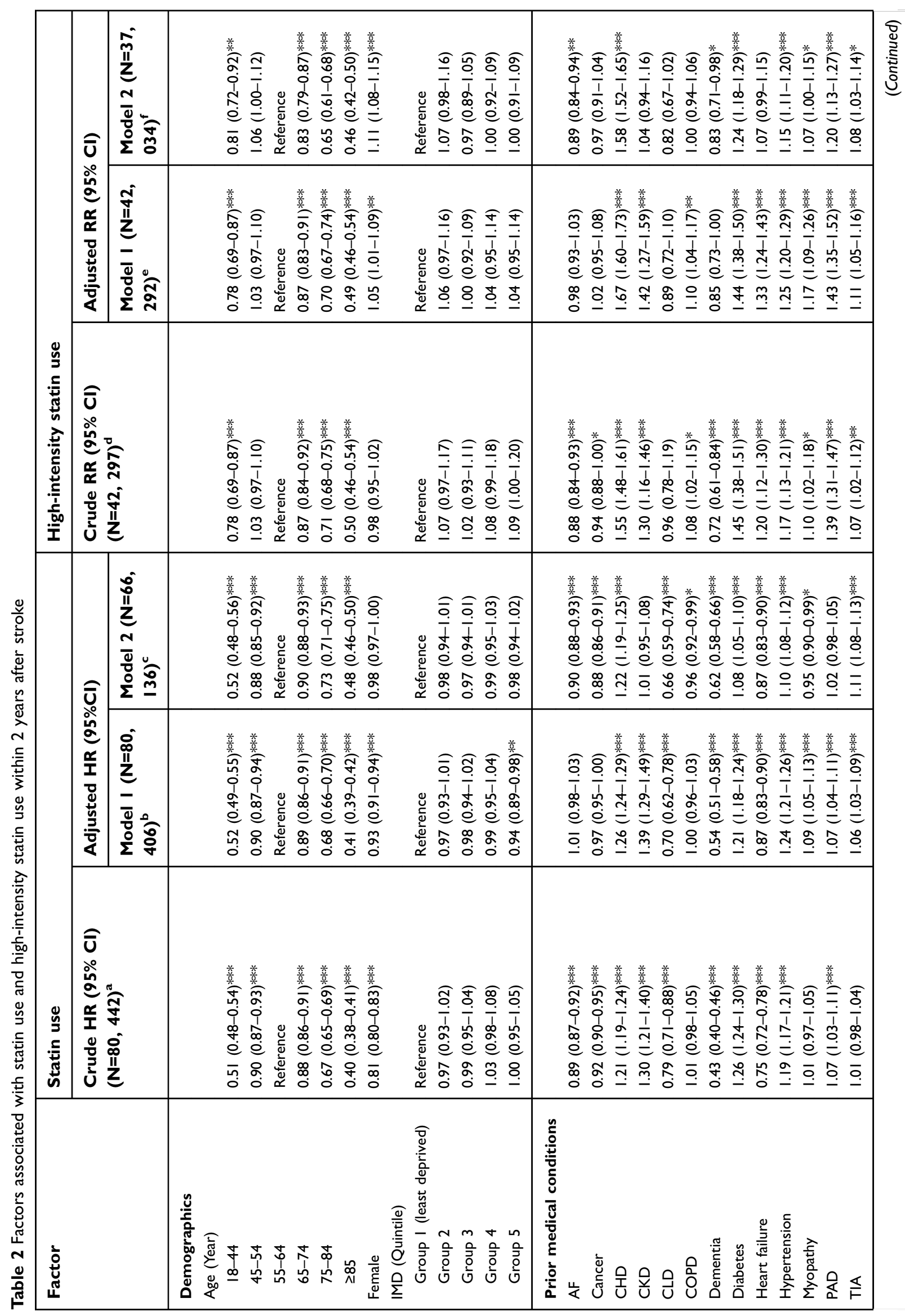




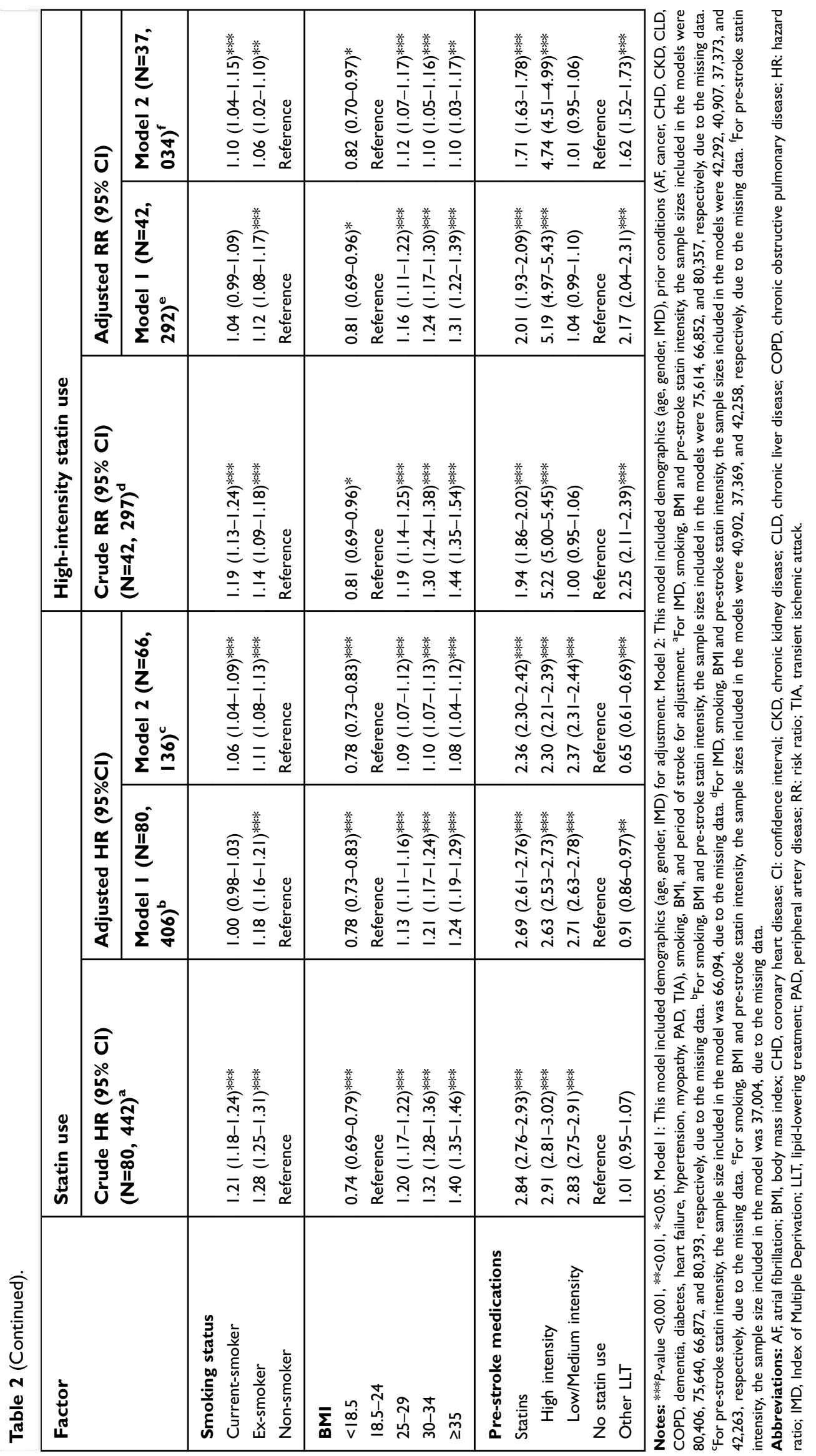


associated with statin use after stroke (Table S6). For those variables that may not meet the proportional hazards assumption (the curves in a $\log$-log plot are not parallel over time) (Figure S2), their associations with statins prescription were strengthened after 90 days of first stroke, except TIA. Sensitivity analysis excluding unspecified stroke conformed to the main analysis, though reduced the power of the analysis (Table S7). When prior statin users were excluded, we observed that CHD, PAD, TIA, and diabetes were associated with decreased probability of statin use after stroke and the association of these cardiovascular comorbidities with high-dose statin use was attenuated (Table S8). A series of sensitivity analyses to cope with missing data did not change the main results, except that 1) the marginally significant associations (including myopathy associated with statin use; dementia, TIA and BMI $\geq 35$ associated with high-intensity statin use) in the main analysis vanished but IMD groups 2 and 3 were associated with slightly decreased use of statins when the analysis was restricted to those with individual IMD; and that 2) the associations of $\mathrm{BMI} \geq 35$ with increased use of any statin and high-intensity statin vanished when the missing value of BMI was replaced by the 95th percentile value.

\section{Discussion}

In this cohort study, we found that statin use after stroke steadily increased between 2000 and 2006, after which time there has been little change. Increased usage was both because more patients were on statins before stroke, and because more patients were initiated on statins after stroke. With regard to high-intensity statins, after some fluctuation from 2000 to 2008 , there has been a steady increase to about $35 \%$ of post-stroke statin users by 2014 .

The changes in statin use after stroke over this time period reflect changes in the clinical evidence and guidelines. Key data on the benefits of statins after ischemic stroke were published between 2000 and 2006. ${ }^{7,8}$ Afterward, the proportion of stroke patients on statins was stable at $70-75 \%$ during the period that targets were in place for UK family doctors. Increasing statin use after ischemic stroke over time has been reported in other studies up until 2010, which focused on use at the time of hospital discharge. ${ }^{24-26}$ Another study using primary care data reported an increase in statin use within 1 year following stroke from $56 \%$ in 2003 to $71 \%$ in $2006 .{ }^{27}$ This study included hemorrhagic stroke, for which the statin use patterns are likely to be different.
Table 3 Factors associated with different chances of using a statin after ischemic stroke, and a high-intensity statin if used

\begin{tabular}{|l|l|l|}
\hline Factor & $\begin{array}{l}\text { Statin use } \\
\text { (relative } \\
\text { percentage } \\
\text { change) }^{\mathbf{a}}\end{array}$ & $\begin{array}{l}\text { High-inten- } \\
\text { sity statin use } \\
\text { (relative per- }^{\text {centage }} \\
\text { change) }^{\mathbf{a}}\end{array}$ \\
\hline
\end{tabular}

Group I: Patients less likely to use statins and less likely to use a high-intensity statin

Age (years) (reference: $55-$

64)

$18-44^{b}$

$65-74$

$75-84$

$\geq 85$

AF

Dementia

No CHD

No diabetes

No hypertension

No TIA

Never smoking

Current smoking

(reference)

Former smoking

(reference)

BMI $<18.5$ (reference: BMI

I8.5-24)

BMI 18.5-24

25-29 (reference)

30-34 (reference)

$\geq 35$ (reference)

No prior statin use

Prior any statin use

(reference)

Prior high-intensity statin

use (reference)

Group 2: Patients less likely to use statins but not less likely to use a high-intensity statin

\begin{tabular}{|l|l|l|}
\hline Age (years) 45-54c (refer- & -12 & +6 \\
ence: 55-64) $^{\text {Cancer }}{ }^{c}$ & -12 & -3 \\
CLD $^{c}$ & -34 & -18 \\
COPD $^{c}$ & -4 & 0 \\
Heart failure $^{c}$ & -13 & +7 \\
Myopathy & -5 & +7 \\
No prior statin use $^{c}$ (reference: & -58 & +1 \\
prior low/medium-intensity $^{\text {statin) }}$ & & \\
Prior non-statin LLT & -35 & +62 \\
\hline
\end{tabular}

(Continued) 
Table 3 (Continued).

\begin{tabular}{|l|l|l|}
\hline Factor & $\begin{array}{l}\text { Statin use } \\
\text { (relative } \\
\text { percentage } \\
\text { change) }^{\mathbf{a}}\end{array}$ & $\begin{array}{l}\text { High-inten- } \\
\text { sity statin use } \\
\text { (relative per- } \\
\text { centage }_{\text {change) }}\end{array}$ \\
\hline $\begin{array}{l}\text { Group 3: Patients not less likely to use statins but less likely } \\
\text { to use a high-intensity statin }\end{array}$ \\
\hline \begin{tabular}{l|l} 
Male \\
No PAD
\end{tabular} & +2 & -10 \\
\hline
\end{tabular}

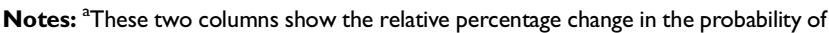
statin and high-intensity statin use compared with a relevant reference group. The percentage in the table was calculated based on the point estimates of HR or RR from the Model 2 (fully adjusted). ' $T$ The two figures in this row, for example, mean patients aged 18-44 years old were $48 \%$ less likely to use statins and 19\% less likely to use high dose statins post-stroke, compared with the reference group of 55-64 years old. 'For patient group 2, these variables were not significantly associated with high-intensity statin use at $P$-value $<0.05$ in Model $2 .{ }^{\mathrm{d}}$ For patient group 3 , these variables were not significantly associated with statin use at $P$-value $<0.05$ in Model 2 .

Abbreviations: $A F$, atrial fibrillation; $B M I$, body mass index; $C H D$, coronary heart disease; CLD, chronic liver disease; COPD, chronic obstructive pulmonary disease; LLT, lipid-lowering treatment; PAD, peripheral artery disease; TIA, transient ischemic attack.

The increase in high-intensity use reflects more recent guidelines, where high-intensity statins have been explicitly recommended for atherosclerotic cardiovascular disease, including ischemic stroke. ${ }^{9-11}$ The availability of generic atorvastatin, whose patent expired in 2011, may have contributed to the increase in the use of high-intensity statins (however, this did not influence the cost to patients, as all UK prescriptions cost a standard charge, from which almost all stroke patients are exempt). Recent studies focusing on CHD suggested that $35 \%$ of patients started high-intensity statins after hospital discharge between 2007 and 2009 and the proportion increased from $33.5 \%$ in 2011 to $71.7 \%$ in 2014. ${ }^{28,29}$ By comparison, statin usage after ischemic stroke in our study is substantially lower. This may be in part attributed to the fact that the risk reduction in CHD achieved by statins is larger than that for stroke. ${ }^{6}$

Our findings of what factors are associated with statin use are consistent with previous reports, ${ }^{24,25,30-33}$ which suggest older age, AF, and dementia are associated with less use of statins after stroke and that hypertension, diabetes, and smoking are associated with increased statin use..$^{24,25,32,34}$ Ours is the first study to explore what factors are associated with high-intensity statin use after stroke. Many of these overlap with the factors associated with low statin use. The most important factor associated with both underuse of statins and lower dose is age, with both older ( $\geq 75$ years) and younger (18-44) patients being less likely to be treated according to current guidelines. This may be justified in older people if a palliative approach is being taken, or reflect concern that the key statin trials in a post-stroke population had mean ages of participants of about 65 years; $;{ }^{7,8}$ underuse may also reflect concerns about the potential increased risk of adverse effects in the presence of multiple comorbidities and polypharmacy. ${ }^{35}$ The underuse of statins in patients with dementia may indicate lack of data on risks and benefits of statins in these patients, who may have reduced ability to adhere to treatment, decreased capacity to experience benefits and increased risk of adverse effects. ${ }^{36}$ The absence of other cardiovascular comorbidities such as diabetes, hypertension, and CKD was consistently associated with lower probability of statin use and high-intensity statin use after stroke, which differs from studies of $\mathrm{CHD}$ and PAD populations. ${ }^{28,29,37}$ This, together with the strong association of statin use (positive association) and other LLT use (inverse association) prior to stroke with post-stroke statin use, suggests that the occurrence of a stroke does not have the impact on statin use that would be expected from the guidelines, ${ }^{9-11}$ and that patients who are new users or who require increased statin doses after stroke may benefit from increased support to overcome clinical inertia. ${ }^{38}$ Paradoxically, patients with CHD, PAD, TIA, or diabetes who were not taking a statin before their stroke were less likely than other patients to initiate a statin after stroke; this may indicate that prior appropriate contraindications to a statin persisted, or that clinical inertia in a high-risk group was not being addressed.

Patients with cancer, heart failure, and non-statin LLT before stroke were at particular risk of not receiving statins. The lower use in heart failure may reflect the negative trials of statins in patients specifically with heart failure, though this would not contraindicate their use after stroke. ${ }^{39-41}$ Patients with CLD were less likely to receive statins. Because all statins are hepatically cleared and can cause liver enzymes elevation, general practitioners may be reluctant to start statin treatment for patients with underlying liver disease given concern about the possible increased risk of hepatotoxicity. ${ }^{20,42}$

While this study has provided some recent data on the trend in and factors for statin use and dose after ischemic stroke, it is still unclear how the adherence to statins post-stroke changes over time and whether the factors associated with the adherence differ between high-dose and low/medium-dose statins. Further evidence in this regard is needed to help improve the secondary prevention of ischemic stroke. 


\section{Strengths and limitations}

Compared with other previous relevant studies, ${ }^{24-27}$ we examined the trend in statin use covering a longer and more up-to-date period and added evidence on the trend in and factors of high-intensity statin use, which is recognized in guidelines as being an important component of preventive treatment after stroke. Our follow-up period of 2 years is an advantage over previous studies, which have only been up to 1 year, ${ }^{24-27}$ since this enabled us to report longer term patterns of statin use, and to confirm that most statin use is initiated within 3 months after the stroke. The study benefits from the strengths of the CPRD in terms of representativeness of real practice settings, detailed prescription information, large sample size, long-term temporal coverage, and sufficient followup duration. ${ }^{18}$

Several limitations of this study should be acknowledged. We had incomplete data regarding the stroke subtypes. Unspecified stroke accounted for more than a half of stroke patients in the CPRD. However, patient characteristics and statin use patterns for these patients were similar to those with ischemic stroke, and sensitivity analysis excluding unspecified stroke did not change the results from the main analysis. Second, missing data also occurred in IMD, smoking status and BMI. Sensitivity analyses using extreme-case approach for smoking and BMI did not change the main results, but restriction to those with complete individual IMD suggested the reverse association of IMD with statin use to reach statistical significance in the more deprived groups. In fact, the association with low socioeconomic status has been observed for access to many aspects of preventive treatment. ${ }^{43,44}$ This suggests that replacing the missing individual IMD with practice-level IMD in our main analysis may dilute the association. Third, some important potential predictors of statin use could not be included, such as lipid profile, patient preference, and practitionerrelated factors, ${ }^{45,46}$ as these data are poorly recorded or not reported in CPRD. Fourth, this study cannot provide any knowledge about the statin use patterns in hospital or at discharge because the CPRD only contains community prescribing information. Fifth, the actual statin use might be overestimated as our analyses were based on the prescription data. It was possible that some patients receiving statins might not actually take them. Finally, this study was based on UK primary care data, which may be of limit applicability to other countries. However, the results we obtained were consistent with those reported elsewhere. ${ }^{24-}$ 26,30-34

\section{Conclusion}

Despite an increase over time in both statin use and high dose use, suboptimal statin treatment remains common in many patients with ischemic stroke. Statin uptake after stroke is lower than after CHD as previously reported, particularly when dose is taken into account. Statin uptake after stroke is also influenced by a variety of pre-stroke cardiovascular comorbidities and LLT. Therefore, there is a need to continue efforts to improve statin utilization and, in particular, increase high-intensity statin use following ischemic stroke. Better understanding of why statins are not taken up as effectively after stroke (eg, through qualitative research) could guide future implementation strategies to improve uptake.

\section{Acknowledgment}

We thank the CPRD@Cambridge team for developing the code lists used in this study.

\section{Disclosure}

The authors report no conflicts of interest in this work.

\section{References}

1. Krishnamurthi RV, Feigin VL, Forouzanfar MH, et al. Global and regional burden of first-ever ischaemic and haemorrhagic stroke during 1990-2010: findings from the global burden of disease study 2010. Lancet Glob Health. 2013;1:e259-e281. doi:10.1016/S2214-109X(13) 70089-5

2. Stroke Association, UK. State of the Nation: stroke statistics. [document on the internet]; 2017. Available from: https://www.stroke.org.uk/ resources/state-nation-stroke-statistics. Accessed November 16, 2018.

3. Lee S, Shafe AC, Cowie MR. UK stroke incidence, mortality and cardiovascular risk management 1999-2008: time-trend analysis from the general practice research database. BMJ Open. 2011;1:e00269. doi:10.1136/bmjopen-2011-000269

4. Koton S, Schneider AL, Rosamond WD, et al. Stroke incidence and mortality trends in us communities, 1987 to 2011. JAMA. 2014;312:259-268. doi:10.1001/jama.2014.7692

5. Bhatnagar P, Scarborough P, Smeeton NC, Allender S. The incidence of all stroke and stroke subtype in the United Kingdom, 1985 to 2008 : a systematic review. BMC Public Health. 2010;10:539. doi:10.1186/ 1471-2458-10-539

6. Manktelow BN, Potter JF. Interventions in the management of serum lipids for preventing stroke recurrence. Cochrane Database Syst Rev. 2009;3:CD002091. doi:10.1002/14651858.CD002091.pub2

7. Collins R, Armitage J, Parish S, Sleight P, Peto R. Effects of cholesterollowering with simvastatin on stroke and other major vascular events in 20536 people with cerebrovascular disease or other high-risk conditions. Lancet. 2004;363:757-767. doi:10.1016/S0140-6736(04)15690-0

8. Amarenco P, Bogousslavsky J, Callahan A 3rd, et al. High-dose atorvastatin after stroke or transient ischemic attack. $N$ Engl $J$ Med. 2006;355:549-559. doi:10.1056/NEJMoa061894 
9. Reiner Z, Catapano AL, De Backer G, et al. ESC/EAS guidelines for the management of dyslipidaemias: the task force for the management of dyslipidaemias of the European Society of Cardiology (ESC) and the European Atherosclerosis Society (EAS). Eur Heart J. 2011;32:1769-1818. doi:10.1093/eurheartj/ehr158

10. Stone NJ, Robinson JG, Lichtenstein AH, et al. ACC/AHA guideline on the treatment of blood cholesterol to reduce atherosclerotic cardiovascular risk in adults: a report of the American College of Cardiology/American Heart Association task force on practice guidelines. Circulation. 2013;2014(129):S1-S45. doi: 10.1161/01. cir.0000437738.63853.7a

11. NICE guideline. Cardiovascular disease: risk assessment and reduction, including lipid modification; 2014. Available from: https://www. nice.org.uk/guidance/cg181/chapter/1-recommendations. Accessed November 16, 2018.

12. National Institute for Health and Care Excellence. Quality and outcomes framework (QOF) indicator development programme; 2013. Available from: https://www.nice.org.uk/media/default/standards-and-indicators/ qof-indicator-assessment-report.Pdf. Accessed November 16, 2018.

13. Kim J, Lee HS, Nam CM, Heo JH. Effects of statin intensity and adherence on the long-term prognosis after acute ischemic stroke. Stroke. 2017;48:2723-2730. doi:10.1161/STROKEAHA.117.018140

14. Chen PS, Cheng CL, Kao Yang YH, Li YH. Statin adherence after ischemic stroke or transient ischemic attack is associated with clinical outcome. Circ J. 2016;80:731-737. doi:10.1253/circj.CJ-15-0753

15. Glader EL, Sjolander M, Eriksson M, Lundberg M. Persistent use of secondary preventive drugs declines rapidly during the first 2 years after stroke. Stroke. 2010;41:397-401. doi:10.1161/ STROKEAHA.109.566950

16. Bushnell CD, Olson DM, Zhao X, et al. Secondary preventive medication persistence and adherence 1 year after stroke. Neurology. 2011;77:1182-1190. doi:10.1212/WNL.0b013e31822f0423

17. Wawruch M, Zatko D, Wimmer G Jr., et al. Patient-related characteristics associated with non-persistence with statin therapy in elderly patients following an ischemic stroke. Pharmacoepidemiol Drug Saf. 2017;26:201-207. doi:10.1002/pds.4148

18. Herrett E, Gallagher AM, Bhaskaran K, et al. Data resource profile: clinical practice research datalink (CPRD). Int $J$ Epidemiol. 2015;44:827-836. doi:10.1093/ije/dyv098

19. Ministry of Housing, Communities \& Local Government. English Indices of Deprivation. London: Gov.Uk.; 2015. Available from: http://www.Gov.Uk/government/statistics/english-indices-of-depriva tion-2015. Accessed November 16, 2018.

20. Collins R, Reith C, Emberson J, et al. Interpretation of the evidence for the efficacy and safety of statin therapy. Lancet. 2016;388:25322561. doi:10.1016/S0140-6736(16)31357-5

21. Cassell A, Edwards D, Harshfield A, et al. The epidemiology of multimorbidity in primary care: a retrospective cohort study. $\mathrm{Br} J$ Gen Pract. 2018;68:e245-e251. doi:10.3399/bjgp18X695465

22. Zou GY, Donner A. Extension of the modified poisson regression model to prospective studies with correlated binary data. Stat Methods Med Res. 2013;22:661-670. doi:10.1177/0962280211427759

23. Ni Chroinin D, Asplund K, Asberg S, et al. Statin therapy and outcome after ischemic stroke: systematic review and meta-analysis of observational studies and randomized trials. Stroke. 2013;44:448456. doi:10.1161/STROKEAHA.112.668277

24. Ovbiagele B, Schwamm LH, Smith EE, et al. Recent nationwide trends in discharge statin treatment of hospitalized patients with stroke. Stroke. 2010;41:1508-1513. doi:10.1161/STROKEA HA.109.573618

25. Sjolander M, Eriksson M, Glader EL. Social stratification in the dissemination of statins after stroke in sweden. Eur $J$ Clin Pharmacol. 2013;69:1173-1180. doi:10.1007/s00228-012-1454-8

26. Grau AJ, Eicke M, Biegler MK, et al. Quality monitoring of acute stroke care in rhineland-palatinate, germany, 2001-2006. Stroke. 2010;41:1495-1500. doi:10.1161/STROKEAHA.110.582239
27. Dregan A, Toschke MA, Wolfe CD, et al. Utility of electronic patient records in primary care for stroke secondary prevention trials. $B M C$ Public Health. 2011;11:86. doi:10.1186/1471-2458-11-86

28. Rosenson RS, Farkouh ME, Mefford M, et al. Trends in use of high-intensity statin therapy after myocardial infarction, 2011 to 2014. J Am Coll Cardiol. 2017;69:2696-2706. doi:10.1016/j. jacc. 2017.03 .585

29. Rosenson RS, Kent ST, Brown TM, et al. Underutilization of high-intensity statin therapy after hospitalization for coronary heart disease. $J$ Am Coll Cardiol. 2015;65:270-277. doi:10.1016/j.jacc.2014.09.088

30. Simpson CR, Wilson C, Hannaford PC, Williams D. Evidence for age and sex differences in the secondary prevention of stroke in scottish primary care. Stroke. 2005;36:1771-1775. doi:10.1161/01. STR.0000173398.99163.9e

31. Asberg S, Henriksson KM, Farahmand B, et al. Ischemic stroke and secondary prevention in clinical practice: a cohort study of 14,529 patients in the swedish stroke register. Stroke. 2010;41:1338-1342. doi:10.1161/STROKEAHA.110.580209

32. Eissa A, Krass I, Bajorek BV. Use of medications for secondary prevention in stroke patients at hospital discharge in australia. Int $J$ Clin Pharm. 2014;36:384-393. doi:10.1007/s11096-013-9908-3

33. Raine R, Wong W, Ambler G, et al. Sociodemographic variations in the contribution of secondary drug prevention to stroke survival at middle and older ages: cohort study. BMJ. 2009;338:b1279. doi:10.1136/bmj.b902

34. Li X, Gao Y, Li J, et al. Underuse of statins in patients with atherosclerotic ischemic stroke in China. Chin Med J (Engl). 2012;125:17031707. doi: 10.3760/cma.j.issn.0366-6999.2012.10.005

35. Catapano AL, Graham I, De Backer G, et al. ESC/EAS guidelines for the management of dyslipidaemias: the task force for the management of dyslipidaemias of the European Society of Cardiology (ESC) and European Atherosclerosis Society (EAS) developed with the special contribution of the European Assocciation for Cardiovascular Prevention \& Rehabilitation (EACPR). Atherosclerosis. 2016;2016 (253):281-344. doi: 10.1016/j.atherosclerosis.2016.08.018

36. Brauner DJ, Muir JC, Sachs GA. Treating nondementia illnesses in patients with dementia. JAMA. 2000;283:3230-3235. doi:10.1001/ jama.283.24.3230

37. Berger JS, Ladapo JA. Underuse of prevention and lifestyle counseling in patients with peripheral artery disease. $\mathrm{J} \mathrm{Am} \mathrm{Coll} \mathrm{Cardiol.}$ 2017;69:2293-2300. doi:10.1016/j.jacc.2017.02.064

38. Phillips LS, Branch WT, Cook CB, et al. Clinical inertia. Ann Intern Med. 2001;135:825-834. doi: 10.7326/0003-4819-135-9-20011106000012

39. Kjekshus J, Apetrei E, Barrios V, et al. Rosuvastatin in older patients with systolic heart failure. $N$ Engl J Med. 2007;357:2248-2261. doi:10.1056/NEJMoa0706201

40. Tavazzi L, Maggioni AP, Marchioli R, et al. Effect of rosuvastatin in patients with chronic heart failure (the gissi-hf trial): A randomised, double-blind, placebo-controlled trial. Lancet. 2008;372:1231-1239. doi:10.1016/S0140-6736(08)61240-4

41. van der Harst P, Voors AA, van Gilst WH, Bohm M, van Veldhuisen DJ. Statins in the treatment of chronic heart failure: biological and clinical considerations. Cardiovasc Res. 2006;71:443-454. doi:10.1016/j.cardiores.2006.04.011

42. Vargas JI, Arrese M, Shah VH, Arab JP. Use of statins in patients with chronic liver disease and cirrhosis: current views and prospects. Curr Gastroenterol Rep. 2017;19:43. doi:10.1007/s11894-017-0584-7

43. Clark AM, DesMeules M, Luo W, Duncan AS, Wielgosz A. Socioeconomic status and cardiovascular disease: risks and implications for care. Nat Rev Cardiol. 2009;6:712-722. doi:10.1038/ nrcardio. 2009.163

44. Cox AM, McKevitt C, Rudd AG, Wolfe CD. Socioeconomic status and stroke. Lancet Neurol. 2006;5:181-188. doi:10.1016/S1474-4422 (06)70351-9 
45. Jamison J, Sutton S, Mant J, De Simoni A. Barriers and facilitators to adherence to secondary stroke prevention medications after stroke: analysis of survivors and caregivers views from an online stroke forum. BMJ Open. 2017;7:e16814. doi:10.1136/bmjopen-2017016814
46. Izuka NJ, Alexander MA, Balasooriya-Smeekens C, Mant J, De Simoni A. How do stroke survivors and their carers use practitioners' advice on secondary prevention medications? Qualitative study of an online forum. Fam Pract. 2017;34:612-620. doi:10.1093/fampra/ cmx023

\section{Publish your work in this journal}

Clinical Epidemiology is an international, peer-reviewed, open access, online journal focusing on disease and drug epidemiology, identification of risk factors and screening procedures to develop optimal preventative initiatives and programs. Specific topics include: diagnosis, prognosis, treatment, screening, prevention, risk factor modification, systematic reviews, risk \& safety of medical interventions, epidemiology \& biostatistical methods, and evaluation of guidelines, translational medicine, health policies \& economic evaluations. The manuscript management system is completely online and includes a very quick and fair peer-review system, which is all easy to use. 